\title{
Application of Solar Energy to Heavy Oil EOR
}

\section{Daniel Palmer, Glass Point}

The presentation will start with an overview of the energy requirements and emissions for thermal EOR and its implications for development of major new heavy oil resources. In addition some fundamentals regarding thermal EOR that are particularly critical for Solar applications will be introduced including steam conditions, water quality requirements and energy storage potential.

The presentation will continue with the specific application of Solar Energy to the development of heavy oil fields, These will include the solar resource potential of key heavy oil regions and the specific requirements for Solar energy as applied to EOR. Economics of the application of Solar compared with gas will be introduced, and the key requirements for favorable economics.

The presentation will finish with a brief case study on the project underway in Oman for PDO. The presentation will conclude with the possibilities for wider application in the Middle East. 